\title{
Intestinal anti-inflammatory effects of RGD- functionalized silk fibroin nanoparticles in trinitrobenzenesulfonic acid-induced experimental colitis in rats
}

\author{
This article was published in the following Dove Press journal: \\ International Journal of Nanomedicine \\ 10 November 2016 \\ Number of times this article has been viewed
}

\author{
Alba Rodriguez-Nogales' \\ Francesca Algieri' \\ Laura De Matteis ${ }^{2}$ \\ A. Abel Lozano-Perez \\ Jose Garrido-Mesa' \\ Teresa Vezza' \\ J M. de la Fuente ${ }^{2}$ \\ Jose Luis Cenis ${ }^{3}$ \\ Julio Gálvez ${ }^{1, *}$ \\ Maria Elena Rodriguez- \\ Cabezas',* \\ 'CIBER-EHD, Department of \\ Pharmacology, ibs.GRANADA, \\ Center for Biomedical Research, \\ University of Granada, Granada, \\ ${ }^{2}$ Instituto de Nanociencia de \\ Aragón, Universidad de Zaragoza, \\ Zaragoza, ${ }^{3}$ Department of \\ Biotechnology, Instituto Murciano de \\ Investigación y Desarrollo Agrario \\ y Alimentario, Murcia, Spain \\ *These authors contributed equally \\ to this work
}

Background: Current treatment of inflammatory bowel disease is based on the use of immunosuppressants or anti-inflammatory drugs, which are characterized by important side effects that can limit their use. Previous research has been performed by administering these drugs as nanoparticles that target the ulcerated intestinal regions and increase their bioavailability. It has been reported that silk fibroin can act as a drug carrier and shows anti-inflammatory properties.

Purpose: This study was designed to enhance the interaction of the silk fibroin nanoparticles (SFNs) with the injured intestinal tissue by functionalizing them with the peptide motif RGD (arginine-glycine-aspartic acid) and to evaluate the intestinal anti-inflammatory properties of these RGD-functionalized silk fibroin nanoparticles (RGD-SFNs) in the trinitrobenzenesulfonic acid (TNBS) model of rat colitis.

Materials and methods: SFNs were prepared by nanoprecipitation in methanol, and the linear RGD peptide was linked to SFNs using glutaraldehyde as the crosslinker. The SFNs (1 mg/rat) and RGD-SFNs ( $1 \mathrm{mg} / \mathrm{rat}$ ) were administered intrarectally to TNBS-induced colitic rats for 7 days.

Results: The SFN treatments ameliorated the colonic damage, reduced neutrophil infiltration, and improved the compromised oxidative status of the colon. However, only the rats treated with RGD-SFNs showed a significant reduction in the expression of different pro-inflammatory cytokines (interleukin [IL]-1 $\beta$, IL-6, and IL-12) and inducible nitric oxide synthase in comparison with the TNBS control group. Moreover, the expression of both cytokine-induced neutrophil chemoattractant-1 and monocyte chemotactic protein-1 was significantly diminished by the RGDSFN treatment. However, both treatments improved the intestinal wall integrity by increasing the gene expression of some of its markers (trefoil factor-3 and mucins).

Conclusion: SFNs displayed intestinal anti-inflammatory properties in the TNBS model of colitis in rats, which were improved by functionalization with the RGD peptide.

Keywords: nanoparticles, inflammatory bowel disease, silk fibroin, TNBS rat colitis, RGD

\section{Introduction}

Ulcerative colitis and Crohn's disease (CD) are considered the two major forms of inflammatory bowel disease (IBD) in humans. They are characterized by chronic gastrointestinal inflammation with discontinuous periods of relapses and remissions. The etiology of this disease remains elusive. Although different theories have been proposed, it is mostly believed that IBD appears in genetically predisposed individuals 
who show an exacerbated immune response in the intestine toward an unknown antigen, most probably derived from the intestinal microbiota. Closely related to this, the sufferers exhibit an altered functionality of the intestinal epithelium barrier that leads to increased permeability to luminal antigens, which easily access the lamina propria and trigger an abnormal immune response that generates the chronic intestinal inflammatory process. ${ }^{1}$ For this reason, restoration of the immune response appears essential for the treatment of IBD patients. In fact, different drug strategies effectively counteract the exacerbated immune response in IBD patients. These include aminosalicylates (sulfasalazine or mesalazine), immunosuppressants (glucocorticoids, azathioprine, methotrexate, and cyclosporine A), and biologicals (infliximab or adalimumab). ${ }^{2}$ However, most of these drug treatments can exert important side effects that may limit their required prolonged use. ${ }^{3}$ For this reason, there is an ongoing, very active search in IBD therapy for new pharmacological strategies that combine intestinal anti-inflammatory effects and safety.

A further improvement of the above-described treatment concept could be the administration of the anti-inflammatory compounds in the form of micro- and nanoparticles. In this regard, Lamprecht et al, ${ }^{4}$ using the trinitrobenzenesulfonic acid (TNBS) model of rat colitis, observed increased accumulation and adhesion of fluorescent polystyrene particles in the ulcerated areas of the affected tissue, with respect to non-ulcerated control tissue. This adhesion was reported to be size dependent, the best results being obtained with $100-\mathrm{nm}$ particles. Considering this, the same group designed a system to transport drugs such as tacrolimus, ${ }^{5}$ low-molecular weight heparin, ${ }^{6}$ and rolipram ${ }^{7}$ on PLGA nanoparticles. Similarly, other studies have assayed the release of dexamethasone from poly-D,L-lactic acid particles for the treatment of TNBS rat colitis ${ }^{8}$ or dextran sodium sulfate-induced intestinal inflammation in mice. ${ }^{9}$ All these studies revealed that the therapeutic effect of the assayed drugs was improved when they were loaded in nanoparticles, most probably due to the targeting to the ulcerated areas. ${ }^{4}$

Nanoparticles of a very different nature could be used as carriers for the delivery of therapeutic compounds. Among them, particles obtained from natural polymers are very interesting since they are biocompatible and biodegradable. ${ }^{10,11}$ An interesting option is the use of silk fibroin nanoparticles (SFNs). Silk fibroin (SF) is the fibrous protein that constitutes the fiber secreted by the silkworm Bombyx mori to make a cocoon for its pupation. This material has many interesting advantages as a scaffold for growing different cell lines, including stem cells. ${ }^{12-14}$ Moreover, SF has been shown to be an excellent matrix for controlled drug delivery, being processed as nano- or microparticles by well-known procedures in aqueous or ionic liquid solution. ${ }^{15,16}$ Fibroin particles have been fabricated by ultrasound vibration, ${ }^{17}$ spray drying, ${ }^{18}$ and desolvation. ${ }^{19,20}$ In addition to its role as a drug carrier, fibroin itself possesses bioactive properties. In this regard, the peptides derived from its hydrolysis have displayed interesting properties; for example, they are able to stimulate glucose transport in both normal and 3T3-L1 insulin-resistant adipocytes ${ }^{21}$ and activate fibroblast proliferation in wound healing models. ${ }^{22}$ The anti-inflammatory properties of fibroin-derived peptides have also been described. Thus, Kim et $\mathrm{al}^{23}$ showed that SF peptides exhibited anti-inflammatory activity in the mice edema model of inflammation induced by topical treatment of mouse ears with 12-O-tetradecanoylphorbol-13-acetate (TPA). This was related to their ability to prevent the increases in the levels of interleukin (IL)-6, IL-1 $\beta$, tumor necrosis factor- $\alpha$, and cyclooxygenase- 2 induced by TPA administration. More recently, Lozano-Pérez et $\mathrm{al}^{24}$ reported that fibroin nanoparticles loaded with resveratrol have intestinal anti-inflammatory effects in the TNBS model of rat colitis, when administered intracolonically. In this study, SFNs administered alone exerted beneficial effects in this experimental model of colitis. In consequence, SFNs represent an interesting option for IBD therapy, since they combine the anti-inflammatory properties of the material together with the possibility of targeting them to the inflamed area. In addition, the effect of fibroin on wound healing, as evidenced in vivo, ${ }^{25}$ may promote its use to treat these pathological intestinal conditions. Moreover, it has been proposed that the molecular mechanisms involved in this effect are related to the ability of fibroin (and also sericin) to induce the up-regulation and phosphorylation of the protein c-Jun that has a role in keratinocyte migration in wound repair. ${ }^{26}$ This could have a positive impact on the altered epithelial barrier function described for human IBD. It is of note that mucosal healing in IBD has been associated with more effective disease control and improved quality of life in these patients, and consequently it has become a key objective in IBD therapy. ${ }^{27}$

Considering all the above, it seems interesting to further improve the intestinal anti-inflammatory properties of SFNs in relation to their potential use in human IBD therapy. With this aim, this study explores the possibility of enhancing the interaction of nanoparticles with the injured tissue, by functionalizing them with the peptide motif RGD 
(arginine-glycine-aspartic acid). The anticipated improvement in the intestinal anti-inflammatory properties exerted by these RGD-functionalized silk fibroin nanoparticles (RGDSFNs) was tested in the TNBS model of rat colitis - a widely used model of colitis that resembles human IBD and in which SFNs have already shown efficacy and a lack of toxicity. ${ }^{24}$ RGD is a well-known motif of adhesion of integrins of the cell surface to the extracellular matrix of connective tissue, which can be covalently bound to the fibroin nanoparticle surface and is extensively used to functionalize different matrices of drug carriers. ${ }^{24,28}$ The rationale of this approach is based on the fact that integrins are highly expressed in human intestinal inflammation, since they are clearly involved in the recruitment of mononuclear cells and leukocyte populations from the bloodstream into the inflamed intestine, and are thus considered to play a critical role in its pathogenesis. ${ }^{29-31}$

\section{Materials and methods Materials}

SF was obtained from silkworms bred in the sericulture facilities of the IMIDA (Murcia, Spain). All other reagents, including the linear RGD peptide (Gly-Arg-Gly-AspSer), were purchased from Sigma-Aldrich Quimica (Madrid, Spain), unless otherwise stated. Purified water $\left(18.2 \mathrm{M} \Omega \cdot \mathrm{cm}\right.$ at $\left.25^{\circ} \mathrm{C}\right)$ was obtained from a Millipore Direct-Q1 ultra-pure water system (EMD Millipore, Billerica, MA, USA). All other chemicals and solvents of analytical grade were used without further purification.

\section{Preparation of reconstituted liquid SF}

Silkworm cocoons were boiled twice for $45 \mathrm{~min}$ in aqueous $0.02 \mathrm{~N} \mathrm{Na}_{2} \mathrm{CO}_{3}$ and rinsed carefully with water to extract the glue-like sericin proteins. Then, the raw SF was dried at room temperature for $72 \mathrm{~h}$. Subsequently, it was dissolved at $10 \%(\mathrm{w} / \mathrm{v})$ in Ajisawa's reagent ${ }^{32}$ - composed of $\mathrm{CaCl}_{2}$ :ethanol: $\mathrm{H}_{2} \mathrm{O}(1: 2: 8$, molar ratio $)$ - for $6 \mathrm{~h}$ at $70^{\circ} \mathrm{C}$. This solution was then dialyzed for $48 \mathrm{~h}$ against distilled water to remove $\mathrm{CaCl}_{2}$, small molecules, and some impurities, using a cellulose semi-permeable membrane (cutoff $3.5 \mathrm{kDa}$ ).

\section{Preparation of SFNs}

The SFNs were prepared by nanoprecipitation in methanol. ${ }^{20}$ In brief, $10 \mathrm{~mL}$ of a $4 \%(\mathrm{w} / \mathrm{v})$ aqueous solution of $\mathrm{SF}$ was dropped into $90 \mathrm{~mL}$ of cold methanol and stirred gently for $2 \mathrm{~h}$. Afterward, the nanoparticles were collected by centrifugation at $18,500 \times g$ for $15 \mathrm{~min}$, at $4^{\circ} \mathrm{C}$, in an Eppendorf $5810 \mathrm{R}$ centrifuge equipped with an F-34-6-38 rotor (Eppendorf, Hamburg, Germany). They were washed three times with ultrapure water, lyophilized, and kept in a desiccator at room temperature until use.

\section{Nanoparticles functionalization with linear RGD peptide \\ Characterization of SFNs previous to \\ functionalization}

The SFNs were characterized in terms of the parameters considered fundamental for a proper design of the following functionalization.

Nanoparticle size was determined in a phosphate-buffered suspension ( $\mathrm{pH}$ 7.4) by dynamic light scattering (DLS) measurement with a Brookhaven 90Plus DLS instrument (Brookhaven Instruments Corporation, Holtsville, NY, USA), by means of photo-correlation spectroscopy. The final data were obtained from a statistical analysis of $\sim 10$ runs.

Field emission scanning electron microscopy (FESEM) was also used to visualize the nanoparticles. A $50 \mu \mathrm{L}$ of SFN suspension (in water) was cast on to a glass wafer, dried overnight, and coated with $\mathrm{Pd}$. The nanoparticles were observed by FESEM using a MERLINTM VP COMPACT scanning electron microscope (Carl Zeiss Microscopy S.L., Oberkochen, Germany). Images detailing the morphology were taken using an SE2 detector, under an accelerating voltage of $15 \mathrm{kV}$ (working distance $=9.3 \mathrm{~mm}$ and aperture size $=30.00 \mu \mathrm{m})$.

In SFNs produced by the method of Zhang et al, ${ }^{33}$ the Lys $\left(\mathrm{CH}_{2}\right)_{4}-\mathrm{NH}_{2}$ groups are exposed on the surface. The nanoparticle amino content was measured by the orange II spectrophotometric assay. ${ }^{34,35}$ Nanoparticles $(1.5 \mathrm{mg})$ were dispersed in $1.5 \mathrm{~mL}$ of $14 \mathrm{mg} / \mathrm{mL}$ orange II acidic solution ( $\mathrm{pH} 3$ ) and maintained under magnetic stirring for $30 \mathrm{~min}$ at $40^{\circ} \mathrm{C}$. The nanoparticles were then precipitated and washed with acidified water until all the unbound dye was removed. Then, an alkaline solution ( $\mathrm{pH}$ 12) was added and the bound dye was desorbed from the surfaces of the nanoparticles. After nanoparticle removal from the medium, the $\mathrm{pH}$ was adjusted to 3 and the amount of desorbed dye was measured at a wavelength of $480 \mathrm{~nm}$, with a Varian Cary $50 \mathrm{UV} /$ Vis spectrophotometer (Agilent Technologies, Inc., Santa Clara, CA, USA).

\section{Functionalization with linear RGD}

The linear RGD peptide was linked to the SFNs using glutaraldehyde as the crosslinker. Briefly, in the first step of the process, $83 \mathrm{nmol}$ of glutaraldehyde was added per mg of SFNs (previously dispersed in $10 \mathrm{mM}$ phosphate buffer at $\mathrm{pH}$ 7.4). The suspension was stirred for 90 min to complete the first coupling reaction. Then, the nanoparticles were centrifuged 
and washed with buffered solution to remove the excess reactant. In the second step of the process, $16 \mathrm{nmol}$ of RGD peptide was added to the glutaraldehyde-activated SFNs and the mixture was stirred for $90 \mathrm{~min}$. Then, the suspension was centrifuged and washed three times with buffered solution. The whole process is summarized in Figure 1.

\section{Evaluation of the efficiency of the functionalization process}

The efficiency of the functionalization process was evaluated by measuring the RGD peptide present in the supernatant of the reaction by the fluorescamine fluorescence spectroscopy assay. ${ }^{36}$ The amount of linked RGD was calculated as the difference between the fluorescence intensity of the control, corresponding to $100 \%$ of the RGD in the reaction mixture, and the fluorescence intensity of the supernatant. The hydrodynamic diameters of the intermediate step of glutaraldehyde functionalization and the final step of RGD-functionalized SFNs (RGD-SFNs) were obtained through DLS measurement, as reported earlier. The data referring to each functionalization step were compared with those referring to the hydrodynamic diameter of the naked
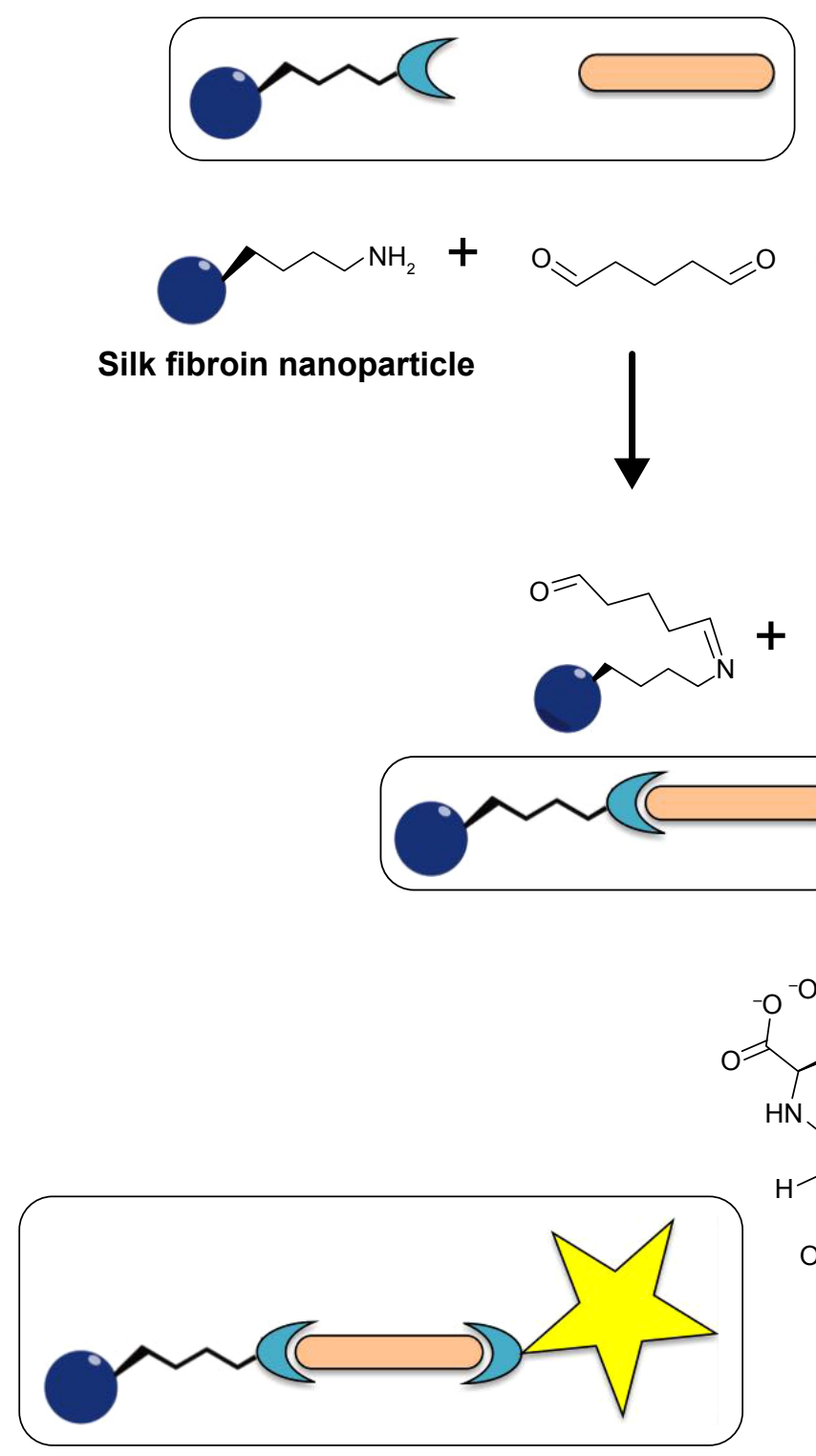

\section{Glutaraldehyde}
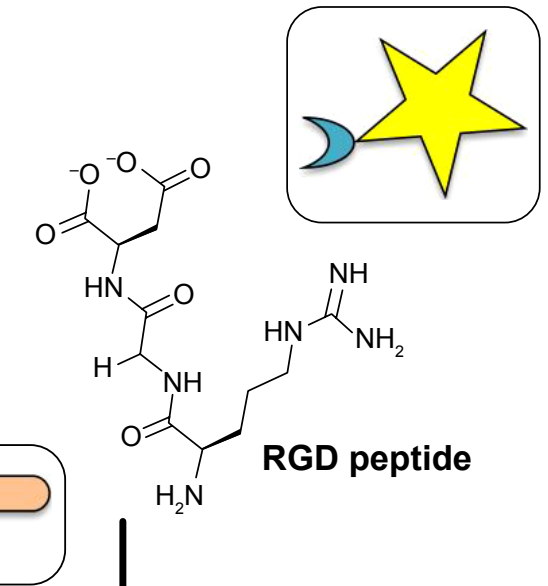<smiles>[R]OCCCCNC(=N)N</smiles>
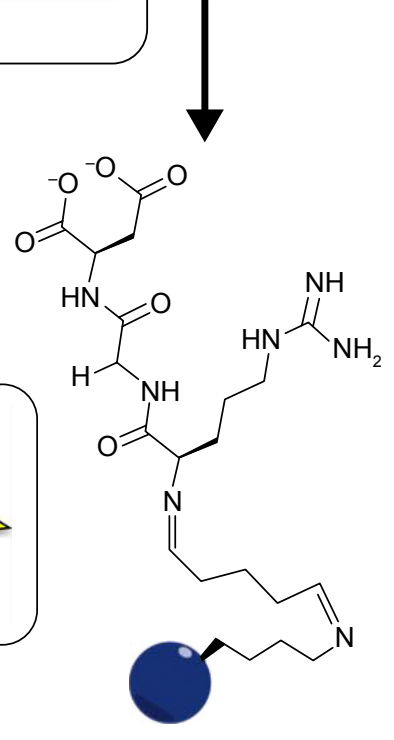

Figure I Functionalization of SFNs with linear RGD peptides through glutaraldehyde crosslinking. Abbreviations: SFNs, silk fibroin nanoparticles; RGD, arginine-glycine-aspartic acid. 
nanoparticles to demonstrate the efficiency of the functionalization process.

\section{In vivo evaluation of the bioactivity of functionalized SFNs in the TNBS model of rat colitis}

This study was performed according to the "Guide for the Care and Use of Laboratory Animals" of the National Institute of Health. The experimental protocol was authorized by the ethics committee on Laboratory Animals of the University of Granada (Spain) (reference number CEEA2010-286). Female Wistar rats (180-200 g) were purchased from Janvier Labs (St Berthevin Cedex, France). They were housed in Makrolon cages and kept in an air-conditioned atmosphere with a 12-h light-dark cycle, with free access to tap water and food. The rats were distributed into four groups $(n=10)$. An untreated TNBS control group and a non-colitic group were included for reference and were given - intrarectally - the same volume of the vehicle used to administer the test products $(0.2 \mathrm{~mL}$ of phosphate-buffered saline). The treatments tested were SFNs (1 mg/rat) and SFNs functionalized with linear RGD (1 mg/rat), in $0.2 \mathrm{~mL}$ of phosphate-buffered saline.

Colonic inflammation was induced in the control and treated groups as described by Camuesco et al. ${ }^{37}$ Briefly, rats were fasted overnight, anesthetized with isoflurane (Isoflo ${ }^{\circledR}$; Esteve, Barcelona, Spain), and $10 \mathrm{mg}$ of TNBS dissolved in $0.25 \mathrm{~mL}$ of $50 \%$ ethanol $(\mathrm{v} / \mathrm{v})$ was instilled by means of a Teflon cannula inserted $8 \mathrm{~cm}$ through the anus. The rats were kept in a head-down position until they recovered from the anesthesia and were put back into their cages. Rats from the non-colitic group received - intrarectally $0.25 \mathrm{~mL}$ of phosphate-buffered saline. The rats were given - intrarectally - the treatments once per day thereafter until being sacrificed with an overdose of isoflurane 7 days later. The animal body weights, occurrence of diarrhea, and water and food intake were recorded daily throughout the experiment. After sacrifice, the colon was removed aseptically, opened, and cleaned of its luminal contents with cold saline. Then, its length and weight were measured. The macroscopic visible damage was scored on a $0-10$ scale by two independent observers, following the criteria described before. ${ }^{37}$ Representative colon samples were taken from an inflamed region adjacent to the gross macroscopic damage, fixed in 4\% buffered formaldehyde, and embedded in paraffin. Equivalent colonic segments were also obtained from the non-colitic group: $5-\mu \mathrm{m}$ paraffin sections were cut at different levels and stained with hematoxylin and eosin. The histologic damage was evaluated by two pathologists blinded for the study following the criteria reported by Arribas et al, ${ }^{38}$ and images were taken by standard light microscopy using an Olympus CX41 microscope (Olympus Iberia, S.A.U., Barcelona, Spain). The colon was subsequently minced, aliquoted, and kept frozen at $-80^{\circ} \mathrm{C}$ until biochemical determinations and RNA extraction were performed.

\section{Evaluation of colonic damage}

Myeloperoxidase (MPO) activity was measured with the technique described by Krawisz et al, ${ }^{39}$ and the results were expressed as MPO units per gram of wet tissue; one unit of MPO activity was defined as that degrading $1 \mu \mathrm{mol}$ of hydrogen peroxide $/ \mathrm{min}$ at $25^{\circ} \mathrm{C}$. The total glutathione (GSH) content was quantified with the recycling assay described by Anderson, ${ }^{40}$ and the results were expressed as nmol/g wet tissue.

\section{Analysis of gene expression in colonic samples by real-time quantitative polymerase chain reaction (RT-qPCR)}

Total RNA from the colonic samples was isolated using TRI Reagent ${ }^{\mathbb{}}$, according to the manufacturer's instructions. After quantifying the RNA, using a Thermo Scientific NanoDrop ${ }^{\text {TM }}$ 2000 spectrophotometer (Thermo Fisher Scientific, Waltham, MA, USA), $2 \mu \mathrm{g}$ of RNA was reverse transcribed using oligo(dT) primers (Promega, Southampton, UK). Subsequently, $20 \mathrm{ng}$ of complementary DNA was taken for real-time quantitative PCR amplification and detection, which was accomplished on optical-grade 48-well plates in an Eco ${ }^{\mathrm{TM}}$ Real-Time PCR System (Illumina, San Diego, CA, USA) with the KAPA SYBR ${ }^{\circledR}$ FAST qPCR Master Mix (Kapa Biosystems, Inc., Wilmington, MA, USA) and specific primers at their annealing temperature (Table 1). The expression of the housekeeping gene glyceraldehyde-3-phosphate dehydrogenase $(G A P D H)$ was measured to normalize messenger RNA (mRNA) expression. The mRNA relative quantitation was calculated with the $\Delta \Delta C t$ method.

\section{Statistics}

All results are expressed as the mean \pm standard error of the mean, except for non-parametric data (scores) - which are expressed as the median (range) and were analyzed using the Mann-Whitney $U$-test. Differences between mean values were tested for statistical significance using a one-way analysis of variance and post hoc least significance tests. All statistical analyses were performed with GraphPad Prism 
Table I Primer sequences used in real-time PCR assays in colonic tissue

\begin{tabular}{|c|c|c|}
\hline Gene & Sequence $5^{\prime}-3^{\prime}$ & $\begin{array}{l}\text { Annealing } \\
\text { temperature } \\
\left({ }^{\circ} \mathrm{C}\right)\end{array}$ \\
\hline \multirow[t]{2}{*}{ GAPDH } & FW: CCATCACCATCTTCCAGGAG & 60 \\
\hline & RV: CCTGCTTCACCACCTTCTTG & \\
\hline \multirow[t]{2}{*}{$I L-I \beta$} & FW: GATCTTTGAAGAAGAGCCCG & 59 \\
\hline & RV: AACTATGTCCCGACCATTGC & \\
\hline \multirow[t]{2}{*}{ IL-6 } & FW: CTTCCAGCCAGTTGCCTTCTTG & 60 \\
\hline & RV: TGGTCTGTTGTGGGTGGTATCC & \\
\hline \multirow[t]{2}{*}{ IL-I 2} & FW: ACGCTACCTCCTCTTCTTG & 60 \\
\hline & RV: ATGTCGTCCGTGGTCTTC & \\
\hline \multirow[t]{2}{*}{ CINC-I } & FW: CCGAAGTCATAGCCACACTCAAG & 60 \\
\hline & RV: TCACCAGACAGACGCCATCG & \\
\hline \multirow[t]{2}{*}{$M C P-I$} & FW: TCTTCCTCCACCACTATGC & 60 \\
\hline & RV: TCTCCAGCCGACTCFATTG & \\
\hline \multirow[t]{2}{*}{ iNOS } & FW: AAGAGACGCACAGGCAGAGG & 59 \\
\hline & RV: AGCAGGCACACGCAATGATG & \\
\hline \multirow[t]{2}{*}{ TFF-3 } & FW: ATGGAGACCAGAGCCTTCTG & 60 \\
\hline & RV: ACAGCCTTGTGCTGACTGTA & \\
\hline \multirow[t]{2}{*}{ MUC-2 } & FW: ACCACCATTACCACCACCTCAG & 60 \\
\hline & RV: CGATCACCACCATTGCCACTG & \\
\hline
\end{tabular}

Abbreviations: PCR, polymerase chain reaction; GAPDH, glyceraldehyde-3phosphate dehydrogenase; IL, interleukin; CINC-I, cytokine-induced neutrophil chemoattractant-I; MCP-I, monocyte chemotactic protein-I; iNOS, inducible nitric oxide synthase; TFF, trefoil factor; MUC, mucin.

version 6.0 (GraphPad Software, Inc., La Jolla, CA, USA), with statistical significance set at $P<0.05$.

\section{Results}

\section{Nanoparticles functionalization with the linear RGD peptide}

\section{Nanoparticle characterization}

The hydrodynamic diameter of the nanoparticles was measured by DLS analysis, and these data were used as the control in the following measurements of the diameter of functionalized nanoparticles. The diameter distribution is reported in Figure 2. The diameter of the nanoparticles varied from 60 to $100 \mathrm{~nm}$, showing a low polydispersity index. The percentage of 450-nm nanoparticles, which were probably produced by aggregation phenomena during the lyophilization process used for nanoparticle storage, was very low. The values are almost identical to those described previously in the literature. . $^{1,33,41}$

The FESEM images of the RGD-SFNs show that the silk particles were globular granules, quite homogeneous in size (Figure 2). As can be seen in the images, no apparent differences in the particle surface were observed, due to the relatively small size of the attached molecules in comparison with the overall size of the nanoparticles.

The orange II spectrophotometric assay was used to determine the amount of amino groups on the nanoparticle surface. This measurement was important in order to adjust the amounts of reactive groups in the functionalization process and to avoid massive crosslinking between nanoparticles. The compound orange II is able to bind to surface amino groups in a $\mathrm{pH}$-dependent way. The method represents an indirect determination of the amino groups since the spectrophotometric analysis is carried out on the compound recovered after breaking such $\mathrm{pH}$-dependent interactions. The advantage of this method is that no interference due to the presence of the nanoparticle is possible. The assay was highly reproducible, giving the proportion of amino groups as $80 \mathrm{nmol} / \mathrm{mg}$ of SFNs.

\section{Nanoparticles functionalization with the linear RGD peptide}

The functionalization process consisted of a two-step protocol, in the first of which the Lys amino groups on the nanoparticle surface were previously functionalized with glutaraldehyde. In the second step, the RGD peptide was added. The RGD peptide possesses a primary amino group that is able to react with glutaraldehyde. All the steps were carried out in $10 \mathrm{mM}$ phosphate buffer ( $\mathrm{pH} 7.4)$, since it allows good stability of the nanoparticle suspension and maintains a low degree of protonation of the reactive amino groups. After the reaction, the functionalized nanoparticles were washed with fresh buffer to eliminate any residual reactant (glutaraldehyde).

The efficiency of the functionalization process was evaluated by measuring the residual peptide in the supernatant of the reaction mixture, with the fluorescamine spectrofluorimetric assay. ${ }^{36}$ The results show that the functionalization protocol led to a peptide linkage efficiency of $65 \%$.

Small changes in the hydrodynamic diameter of the nanoparticles were apparent from the DLS measurements made after every reaction step, confirming the successful binding of the biomolecules through the glutaraldehyde crosslink. The changes in the size distribution of the nanoparticles during the process are exhibited in Figure 2. The size distribution was not changed significantly by the first step of the process. In contrast, Figure 2 shows how it changed after peptide binding: the increase in the hydrodynamic diameter of the nanoparticles constitutes evidence of the efficiency of the functionalization process.

Also, the Z-potential of the nanoparticles before and after functionalization was measured at different $\mathrm{pH}$ values to better characterize the changes in the surface after RGD binding. In Figure 2F, a significant difference in the Z-potential can be observed between the synthesized SFNs and the 


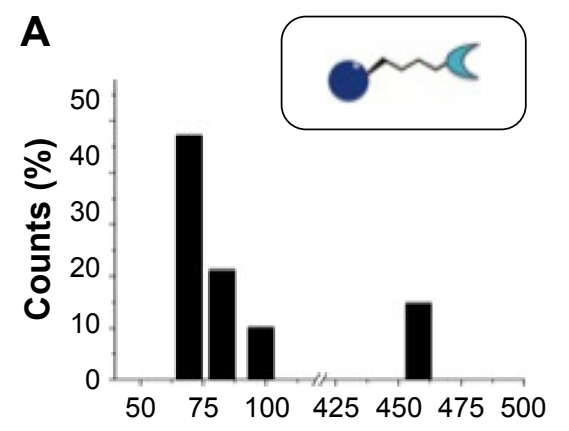

Hydrodynamic diameter ( $\mathrm{nm}$ )

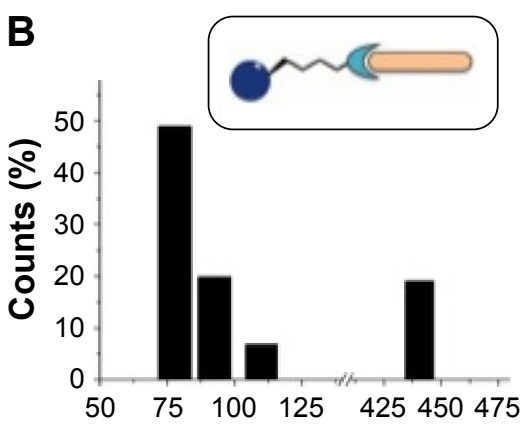

Hydrodynamic diameter $(\mathrm{nm})$

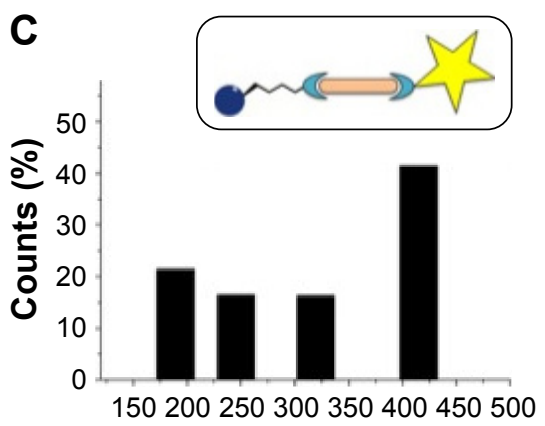

Hydrodynamic diameter (nm)
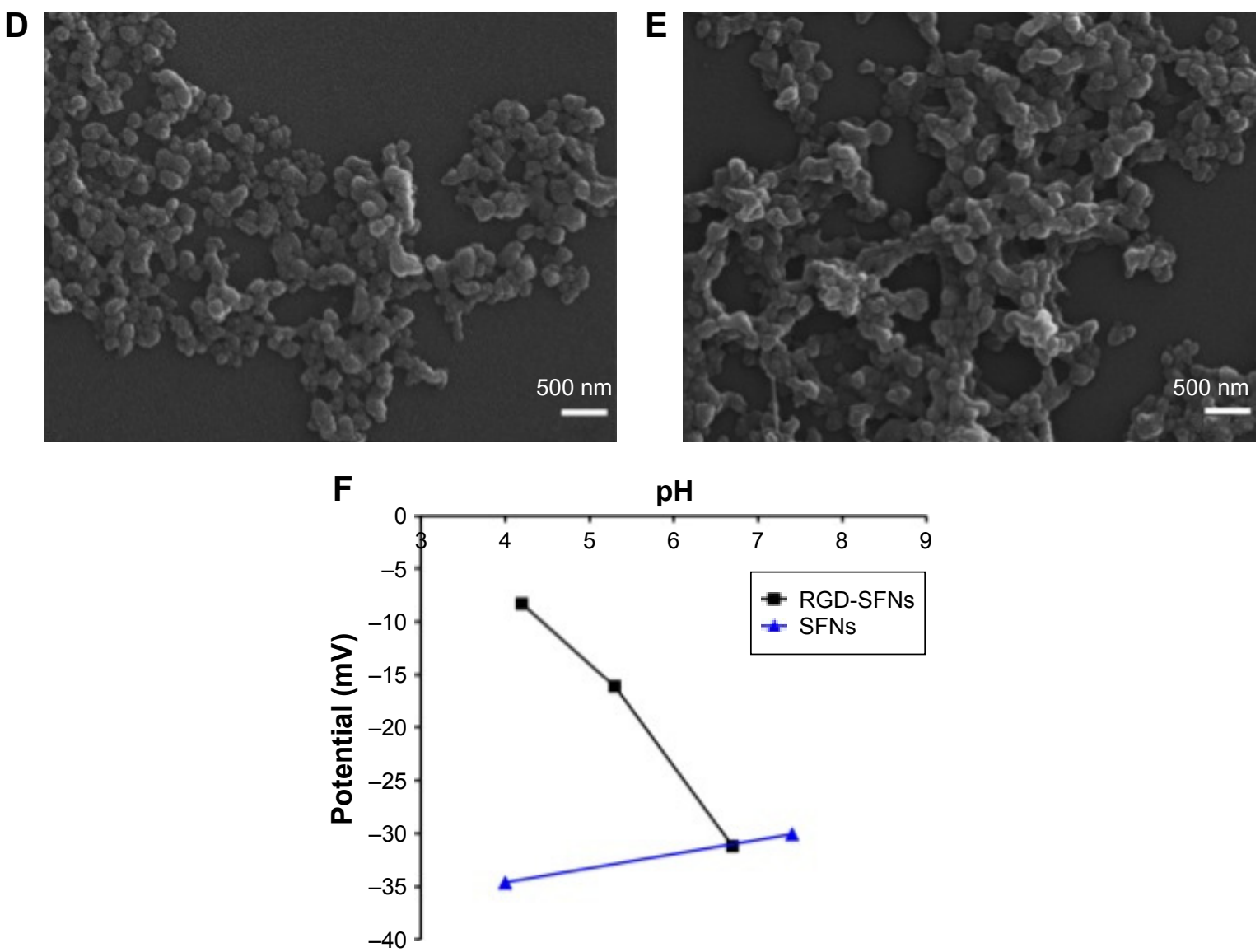

Figure 2 Morphological characterization of the nanoparticles.

Notes: Hydrodynamic diameter distribution of (A) naked SFNs before functionalization; (B) glutaraldehyde-activated SFNs; and (C) SFNs with RGD bound on the surface (the insets represent the surface modifications). FESEM images of (D) naked SFNs before functionalization and (E) SFNs with RGD bound on the surface (scale bar $500 \mathrm{~nm}$, magnification 17.25 K). (F) Z-potential analysis of SFNs and RGD-SFNs.

Abbreviations: SFNs, silk fibroin nanoparticles; RGD, arginine-glycine-aspartic acid; FESEM, field emission scanning electron microscopy; RGD-SFNs, RGD-functionalized SFNs.

RGD-functionalized particles (especially at acidic $\mathrm{pH}$ values), due to the presence of the RGD molecules on the surface.

\section{Intestinal anti-inflammatory activity of RGD-SFNs}

The objective of this assay was to examine if the functionalization of the SFNs resulted in an additional antiinflammatory effect when compared with the bare nanoparticles. The results show that both types of SFNs displayed intestinal anti-inflammatory effects. This was demonstrated macroscopically since the colonic weight/ length ratio was significantly reduced in comparison with the colitic control group (Table 2), suggesting a reduction of the edema related to the TNBS-induced inflammatory process. Moreover, the two treatments also produced a significant decrease in the extension of the damaged areas in the colon, 
Table 2 Effects of RGD-SFNs (I mg/rat, intrarectally) on the colonic macroscopic damage score, weight/length ratio, MPO activity, and GSH content in TNBS experimental rat colitis

\begin{tabular}{lllll}
\hline Group $(\mathbf{n}=\mathbf{1 0})$ & Damage score $(\mathbf{0}-\mathbf{1 0})$ & Weight/length $(\mathbf{m g} / \mathbf{c m})$ & MPO $(\mathbf{m U} / \mathbf{g}$ tissue) & GSH (nmol/g tissue) \\
\hline Non-colitic & $0^{\mathrm{a}}$ & $66.2 \pm 4.4^{\mathrm{a}}$ & $18.7 \pm 5.9^{\mathrm{a}}$ & $2076 \pm 86^{\mathrm{a}}$ \\
Colitic control & $7.5(6-8)^{\mathrm{b}}$ & $228.4 \pm 16.1^{\mathrm{b}}$ & $230.3 \pm 23.4^{\mathrm{b}}$ & $791 \pm 66^{\mathrm{b}}$ \\
SFNs & $5.0(4-8)^{\mathrm{c}}$ & $158.9 \pm 31.2^{\mathrm{c}}$ & $143.0 \pm 27.3^{\mathrm{c}}$ & $1487 \pm 26 \mathrm{I}^{\mathrm{c}}$ \\
RGD-SFNs & $5.5(4-7)^{\mathrm{c}}$ & $146.1 \pm 29.6^{\mathrm{c}}$ & $49.9 \pm 10.7^{\mathrm{d}}$ & $119| \pm| 88^{\mathrm{c}}$ \\
\hline
\end{tabular}

Notes: Score data are expressed as the median (range) and the rest of the data as the mean \pm SEM. Groups with different letters differ statistically $(P<0.05)$.

Abbreviations: RGD, arginine-glycine-aspartic acid; SFNs, silk fibroin nanoparticles; RGD-SFNs, RGD-functionalized silk fibroin nanoparticles; MPO, myeloperoxidase; GSH, glutathione; TNBS, trinitrobenzenesulfonic acid; SEM, standard error of the mean.

since the treated groups had lower values of the macroscopic score than the control colitic group (Table 2). Hence, the administration of nanoparticles facilitated the recovery of the damaged colon. This observation was confirmed after the histologic evaluation. The TNBS produced a significant disruption of the colonic mucosa - affecting $>80 \%$ of the surface, involving all the layers - and was characterized by epithelial ulceration, crypt hyperplasia, and intense goblet cell depletion. Severe granulocyte infiltration was observed, mainly involving neutrophils in the lamina propria. The grade of the lesion was scored as severe or very severe (31 [20-46]) (Figure 3). Both SFN treatments significantly enhanced the intestinal cytoarchitecture by lessening the damaged surface area - to $\sim 25 \%$ - and the transmural involvement, thus improving the reepithelialization. In addition, the inflammatory infiltrate was slight to moderate with a patchy distribution and the goblet cells appeared to be in the process of recovery; some of them had replenished their mucin content. Therefore, the microscopic scores assigned to these groups were significantly lower than for the control colitic group (14 [8-20] [SFNs] and 14 [4-22] [RGD-SFNs], $P<0.05$ ) (Figure 3).

The biochemical parameters evaluated in the colon confirmed the intestinal anti-inflammatory effect exerted by both types of SFNs, bare or functionalized with RGD. Thus, MPO activity was significantly reduced in the treated colitic groups when compared with control colitic rats, probably due to the significant reduction of the leukocyte infiltration into the inflamed intestinal tissue that was observed and that characterizes this experimental model of colitis ${ }^{37}$ (Table 2). However, for the colitic group that received the RGD-SFNs, the reduction in this enzyme activity was greater than that obtained with the bare nanoparticles (Table 2). TNBS colitis is also characterized by an intense oxidative insult that depletes the antioxidant peptide GSH, ${ }^{37,42,43}$ but the nanoparticles treatments managed to increase the colonic GSH content, thus diminishing the severity of the oxidative stress that characterizes the inflamed colon (Table 2).
The colonic expression of the pro-inflammatory cytokines IL-1 $\beta$, IL-6, and IL-12 was significantly greater in the colitic groups than in the non-colitic rats, indicating an altered immune response induced by the instillation of TNBS (Figure 4). However, only the colitic rats treated with RGDSFNs showed a significant reduction in the expression of these three cytokines in comparison with colitic rats from the control group or those that received the bare SFNs (Figure 4).

Colonic inflammation was also associated with an increased expression of the chemokine cytokine-induced neutrophil chemoattractant (CINC)-1, which promotes the recruitment and later activation of neutrophils in the inflamed area, and of the monocyte chemotactic protein (MCP)-1 that favors the chemotaxis of monocytes and their subsequent differentiation into macrophages in the inflamed tissue (Figure 5). Similarly to the previous result, only the RGD-SFNs managed to significantly reduce the expression of both CINC-1 and MCP-1 in comparison with the colitic control group (Figure 5).

Moreover, TNBS instillation also resulted in an increased colonic expression of iNOS, in accordance with previous results obtained in experimental models of rodent colitis. ${ }^{44,45}$ Again, it is of note that only the colitic rats treated with RGD-SFNs showed a significantly reduced expression of colonic iNOS when compared with the control colitic groups, whereas the bare nanoparticles only produced a tendency of the expression to decrease, but no statistical differences were found (Figure 5).

Finally, when different markers related to the integrity of the intestinal epithelium were evaluated, the TNBS intestinal damage was characterized by reduced expression of the intestinal trefoil factor (TFF)-3, a bioactive peptide involved in epithelial protection and repair, and the mucin MUC-2, the primary constituent of the mucus layer in the colon (Figure 6) ${ }^{46}$ Both treatments with SFNs (RGD functionalized and bare) increased the expression of TFF- 3 and MUC-2 (Figure 6), thus contributing to the restoration of the epithelial barrier and the preservation of the mucus layer that covers 
A

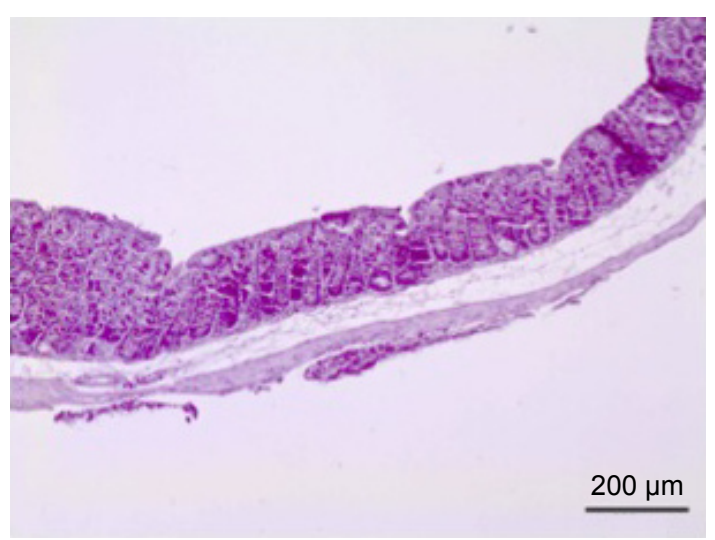

C

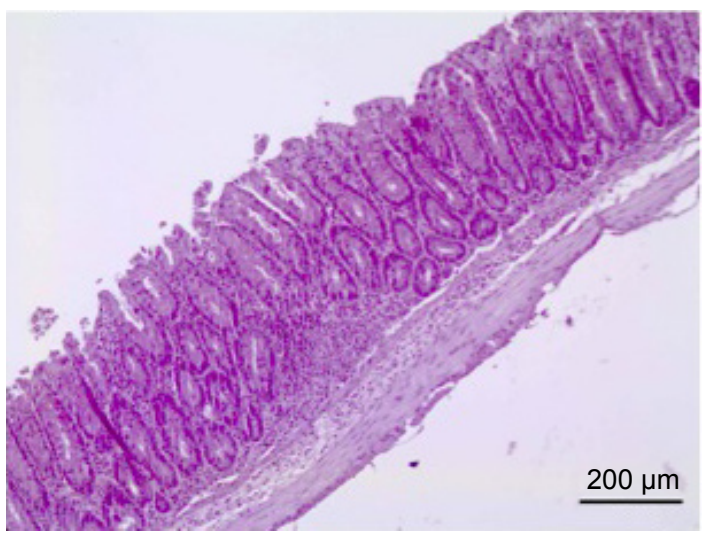

B

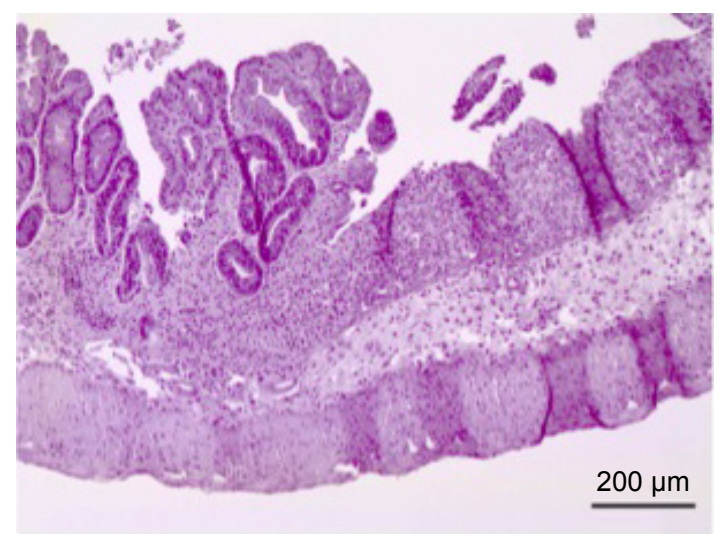

D

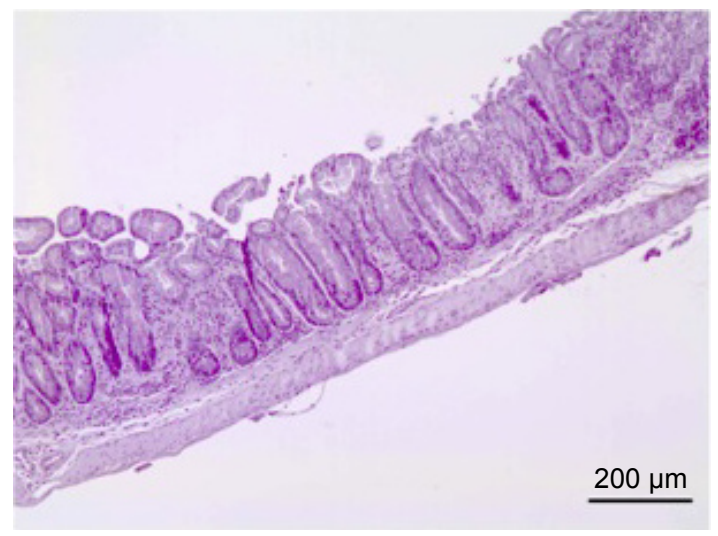

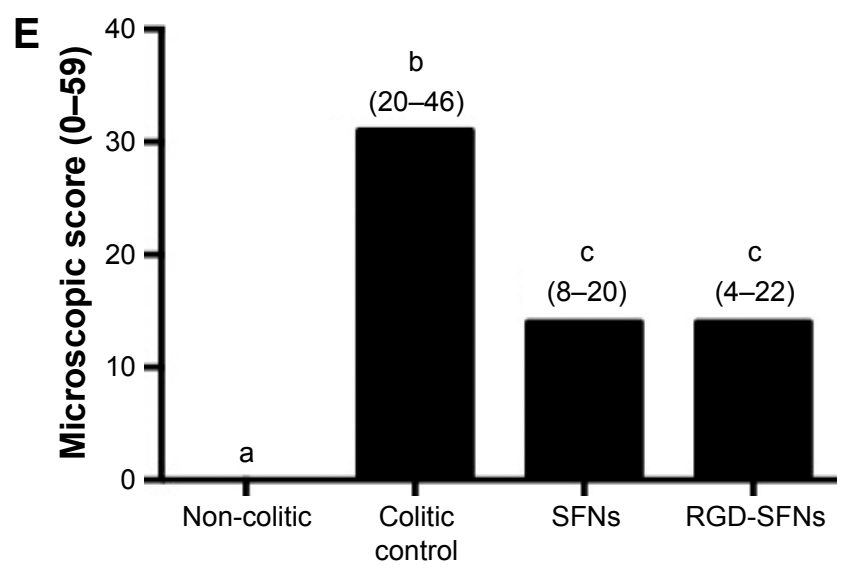

Figure 3 Histologic sections of colon stained with hematoxylin and eosin.

Notes: (A) Non-colitic; (B) colitic control; (C) SFNs; and (D) RGD-SFNs. (E) Microscopic score assigned according to the criteria previously described. ${ }^{38}$ The data are expressed as the median (range); groups with different letters differ statistically $(P<0.05)$.

Abbreviations: SFNs, silk fibroin nanoparticles; RGD, arginine-glycine-aspartic acid; RGD-SFNs, RGD-functionalized SFNs.

and protects the integrity of the epithelium and accelerating the recovery of the damaged intestinal mucosa.

\section{Discussion}

Currently, there is a demand for new therapeutic strategies for human IBD. Although many different drug treatments with reputed efficacy against this intestinal condition are available, most of them are not devoid of significant side effects that limit their use - especially when they are prescribed chronically, as required for most patients. Moreover, complicated dosing schedules and a somewhat excessive cost can also make their long-term usage problematic. ${ }^{3}$ A previous study with the TNBS experimental model of rat colitis demonstrated that SFNs could be used as carriers for the controlled release of active drugs, such as resveratrol, used against intestinal inflammation. ${ }^{24}$ In the same study, the bare SFNs also exerted beneficial effects when administered intrarectally, showing an efficacy similar to that 


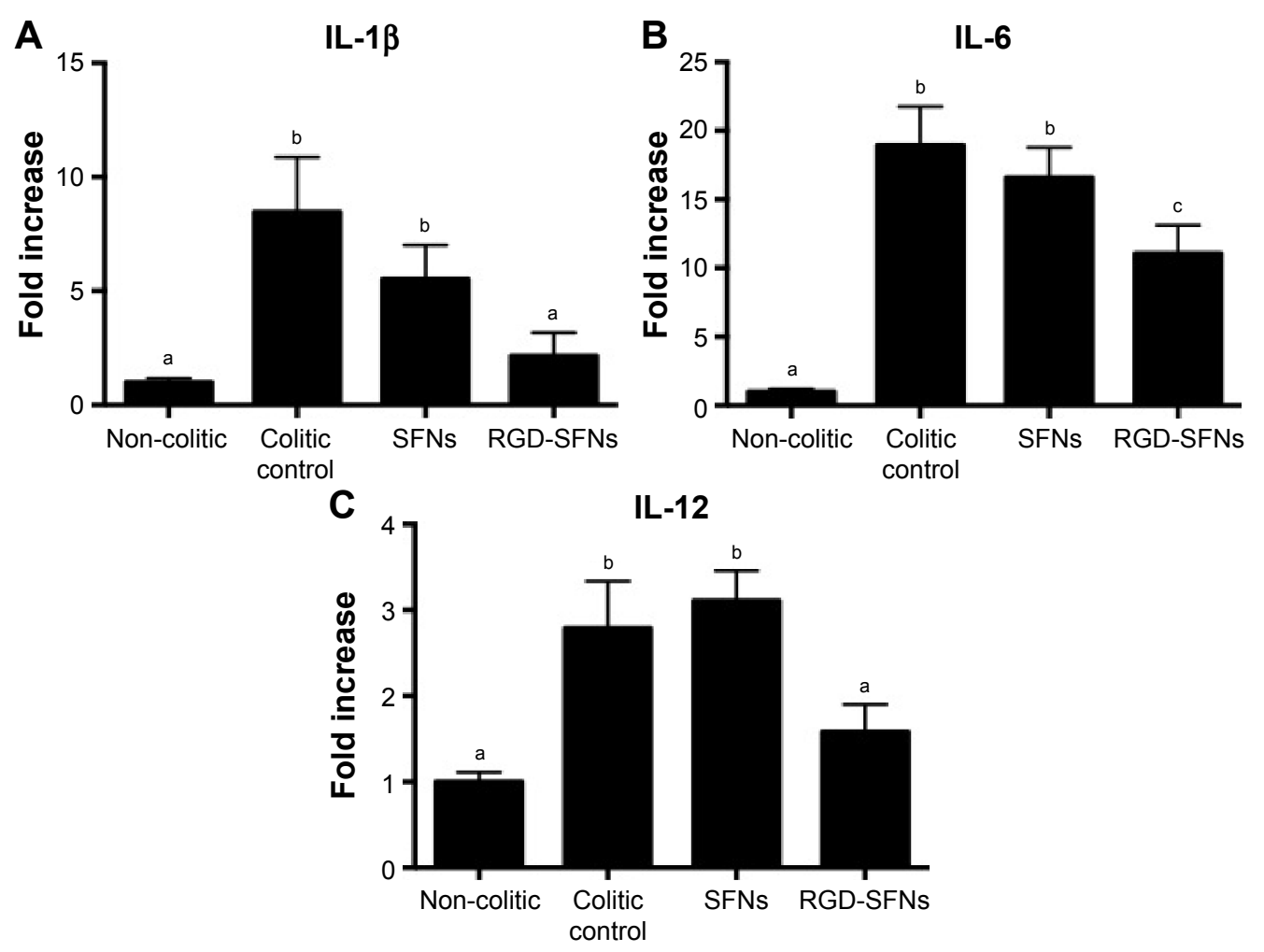

Figure 4 Effects of RGD-SFNs (I mg/rat, intrarectally) in TNBS rat colitis on colonic gene expression of the cytokines (A) IL-I $\beta$, (B) IL-6, and (C) IL-I2, analyzed by real-time PCR.

Notes: The data are expressed as the mean \pm SEM. Groups with different letters differ statistically $(P<0.05)$.

Abbreviations: RGD, arginine-glycine-aspartic acid; SFNs, silk fibroin nanoparticles; RGD-SFNs, RGD-functionalized SFNs; TNBS, trinitrobenzenesulfonic acid; IL, interleukin; PCR, polymerase chain reaction; SEM, standard error of the mean.

of dexamethasone, a glucocorticoid that is currently used in human IBD therapy. ${ }^{2}$ In consequence, fibroin nanoparticles should not be considered just as inert carriers when used in intestinal inflammation therapy. Furthermore, it has been demonstrated that SFNs have the special ability to target ulcerated and damaged areas of the intestine, ${ }^{4}$ which could contribute to their beneficial local effects on these intestinal conditions. So, the design of additional strategies to enhance their intestinal anti-inflammatory properties is of great interest. In this way, the drugs could be used at lower doses while keeping/enhancing their efficacy, but improving their pharmacological profile and reducing the side effects.

With this aim, SFNs were functionalized with RGD, a motif of adhesion of integrins of the cell surface to the extracellular matrix of connective tissue. It has been proposed that integrins may play a key role in the pathogenesis of IBD, since they are highly expressed in the gut of IBD patients and are involved in leukocyte recruitment to the inflamed intestinal tissue. ${ }^{29}$ In fact, they have been considered an interesting target for IBD treatment, and antibodies directed to integrins, such as natalizumab and vedolizumab, have shown efficacy in human clinical trials. ${ }^{47}$
Nanoparticle functionalization with the RGD peptide was successfully carried out using glutaraldehyde as the homobifunctional crosslinker. This reaction was carefully optimized in terms of the reactant/SFNs ratio. In this way, no aggregation due to nanoparticles crosslinking was observed.

The SFNs possess a very negatively charged surface in their naked state (before functionalization). The shift of the surface potential to less negative values, found after functionalization, indicates that the adhesive properties of the material per se could be enhanced by a masking of the negative charge due to the presence of the peptide.

This study confirms the intestinal anti-inflammatory properties of the SFNs in the TNBS model of experimental colitis, as evidenced both macroscopically and biochemically, even at $1 \mathrm{mg} / \mathrm{rat}$, a dose lower than that used in the previous study $(8 \mathrm{mg} / \mathrm{rat}){ }^{24}$ However, the incorporation of RGD into the SFNs resulted in beneficial effects additional to those shown by bare SFNs, as demonstrated when the colonic tissue was evaluated biochemically. In this regard, colonic MPO activity is related to neutrophil infiltration and has long been used as a marker of the severity of the intestinal inflammatory 


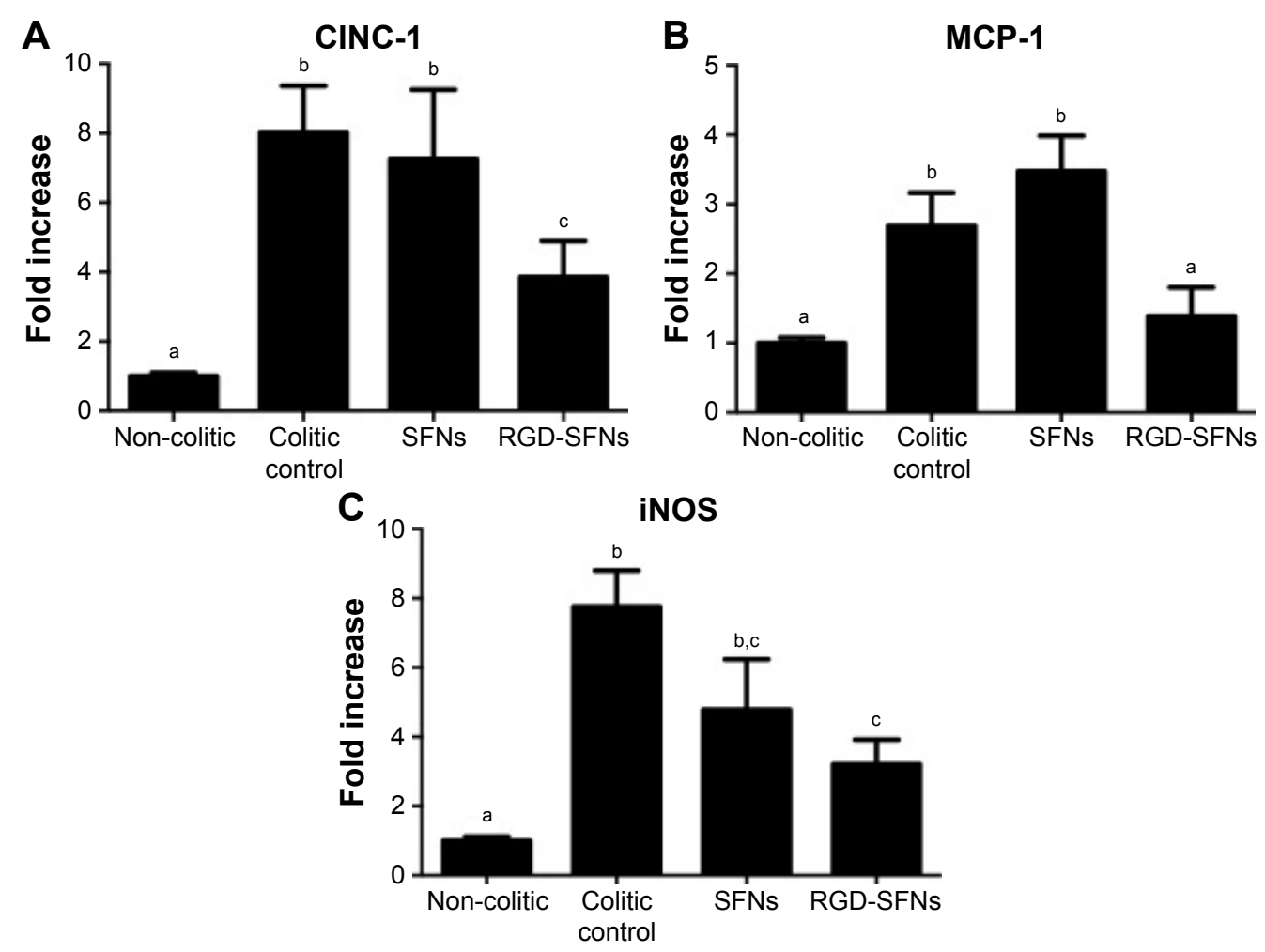

Figure 5 Effects of RGD-SFNs (I mg/rat, intrarectally) in TNBS rat colitis on colonic gene expression of the chemokines (A) CINC-I and (B) MCP-I and of (C) the inducible enzyme iNOS, analyzed by real-time PCR.

Notes: The data are expressed as the mean \pm SEM. Groups with different letters differ statistically $(P<0.05)$.

Abbreviations: RGD, arginine-glycine-aspartic acid; SFNs, silk fibroin nanoparticles; RGD-SFNs, RGD-functionalized SFNs; TNBS, trinitrobenzenesulfonic acid; IL, interleukin; CINC-I, cytokine-induced neutrophil chemoattractant-I; MCP-I, monocyte chemotactic protein-I; iNOS, inducible nitric oxide synthase; PCR, polymerase chain reaction; SEM, standard error of the mean.

process. ${ }^{44,48}$ Supporting this, intestinal inflammation has been widely proposed to start with leukocyte infiltration into the lamina propria, which facilitates the chronicity of the inflammatory response. ${ }^{49}$ For this reason, inhibition of MPO activity in the colon can be considered as a manifestation of the intestinal anti-inflammatory effect of a given treatment. ${ }^{50}$ Thus, in this study, when MPO activity was assayed in the colon of colitic rats, the lowest values were observed in rats treated with RGD-SFNs, being statistically different from the values obtained with SFNs $(P<0.05)$. This agrees with the
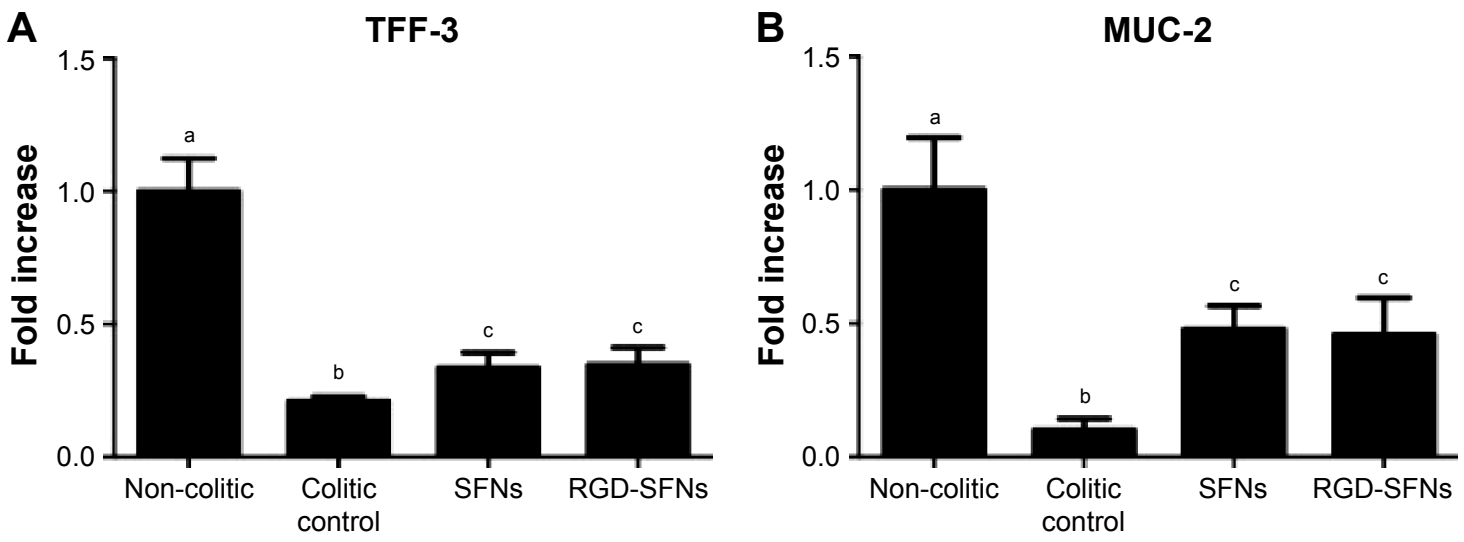

Figure 6 Effects of RGD-SFNs (I mg/rat, intrarectally) in TNBS rat colitis on colonic gene expression of (A) the trefoil factor TFF-3 and (B) the mucin MUC-2. Notes: The data are expressed as the mean \pm SEM. Groups with different letters differ statistically $(P<0.05)$.

Abbreviations: RGD, arginine-glycine-aspartic acid; SFNs, silk fibroin nanoparticles; RGD-SFNs, RGD-functionalized SFNs; TNBS, trinitrobenzenesulfonic acid; PCR, polymerase chain reaction; SEM, standard error of the mean. 
histologic evaluation, although no statistical differences were observed between the two treated groups $(P>0.05)$. The higher efficacy could be attributed to the differences detected when the chemokines CINC-1 and MCP-1 were evaluated. In fact, only the treatment with RGD-SFNs was able to significantly decrease their expression in the inflamed colon. CINC-1, a functional analog of IL-8, is involved in neutrophil recruitment and activation. ${ }^{51}$ Similarly, the chemokine MCP-1 contributes to the inflammatory process by promoting the recruitment to sites of injury of monocytes, memory T-cells, dendritic cells, and, indirectly, neutrophils. ${ }^{52}$ Moreover, the significant reduction in the expression of MCP-1 could account for the lower infiltration and the subsequent amelioration of the macrophage activation of the colon damage. Furthermore, this may be related to a reduced expression of the inducible enzyme iNOS, which was only seen in the RGD-SFN-treated group, since macrophages are considered one of the main sources of iNOS expression in inflammatory conditions. ${ }^{45}$

The greater inhibitory effect exerted by the RGD-SFNs on leukocyte infiltration could also explain the differences observed in the pro-inflammatory cytokines IL-1 $\beta$, IL-6, and IL-12 among the groups, thus revealing an improvement in the altered immune response. It is of note that the expression of each of these cytokines has been found to be up-regulated in human IBD, their effects on the intestine being closely associated with the generation of colonic damage. ${ }^{53}$

Both treatments resulted in a partial amelioration of some biochemical markers related to epithelial integrity, which could also effectively account for their intestinal anti-inflammatory effect. Thus, both treatments significantly increased the expression of the mucin MUC-2, one of the major components of the intestinal mucus layer, ${ }^{54}$ and of TFF-3, one of the peptides involved in epithelial protection and repair. ${ }^{55}$ In consequence, the development of the mucus-secreting layer that covers the epithelium and acts as a physical barrier may have protected its integrity and accelerated the recovery of the injured colonic tissue, which was also appreciated in the histologic evaluation. In this regard, it has been proposed that one of the early events in gut inflammation is a defect in the epithelial barrier function, which promotes the entry of luminal antigens that facilitate an exaggerated immune response. So, the speeding-up of mucosal restoration appears to be of special significance. In fact, healing of the inflamed mucosa is now emerging as a key goal and a tool to predict clinical success in the management of IBD. ${ }^{56}$

\section{Conclusion}

Fibroin nanoparticles have been used successfully in this study as carriers for the RGD peptide. The functionalization process has been optimized, and covalent binding - through glutaraldehyde crosslinking - with a linear RGD peptide has been obtained. These nanocarriers have been tested and compared in the treatment of intestinal inflammation in rats. The treatments involving the daily administration of fibroin nanoparticles by the intrarectal route, at a dose of $1 \mathrm{mg} / \mathrm{rat}$, showed a beneficial effect in the model of experimental colitis induced by TNBS in rats. When considering the impact on the altered immune response that characterizes the intestinal inflammatory process, this effect was clearly improved when the nanoparticles were functionalized with RGD.

\section{Acknowledgments}

This work was supported by the Junta de Andalucia (P10AGR-6826 and CTS 164) and the Spanish Ministry of Economy and Competitiveness (AGL2015-67995-C3-3-R) with funds from the European Union, Fondo Social de la DGA (grupos DGA), Ministerio de la Economía y Competitividad del Gobierno de España for the public funding of Proyectos I+D+I - Programa Estatal de Investigación, Desarrollo e Innovación Orientada a los Retos de la Sociedad (SAF2014-54763-C2-2-R), and CASEN-RECORDATI S.A. A Rodriguez-Nogales and F Algieri are postdoctoral fellows of the University of Granada; AA Lozano-Perez's research contract is partially supported $(80 \%)$ by the FEDER/ ERDF Program of the Region of Murcia 2014-2020 (FEDER 14-20-01). ME Rodriguez-Cabezas is a postdoctoral fellow of CIBER-EHD. The CIBER-EHD is funded by the Instituto de Salud Carlos III. We thank SD Aznar-Cervantes, for his technical assistance in the production of SFNs, and N Garrido-Mesa and MP Utrilla, for their contributions to the in vivo biological evaluation of the effect of SFNs.

\section{Author contributions}

All authors contributed toward data analysis, drafting and critically revising the paper and agree to be accountable for all aspects of the work.

\section{Disclosure}

The authors report no conflicts of interest in this work.

\section{References}

1. Xavier RJ, Podolsky DK. Unravelling the pathogenesis of inflammatory bowel disease. Nature. 2007;448(7152):427-434.

2. Bernstein CN. Treatment of IBD: where we are and where we are going. Am J Gastroenterol. 2015;110(1):114-126. 
3. Siegel CA. Review article: explaining risks of inflammatory bowel disease therapy to patients. Aliment Pharmacol Ther. 2011;33(1):23-32.

4. Lamprecht A, Schafer U, Lehr CM. Size-dependent bioadhesion of micro- and nanoparticulate carriers to the inflamed colonic mucosa. Pharm Res. 2001;18(6):788-793.

5. Lamprecht A, Yamamoto H, Takeuchi H, Kawashima Y. Nanoparticles enhance therapeutic efficiency by selectively increased local drug dose in experimental colitis in rats. J Pharmacol Exp Ther. 2005;315(1): 196-202.

6. Pellequer Y, Meissner Y, Ubrich N, Lamprecht A. Epithelial heparin delivery via microspheres mitigates experimental colitis in mice. J Pharmacol Exp Ther. 2007;321(2):726-733.

7. Lamprecht A, Ubrich N, Yamamoto H, et al. Biodegradable nanoparticles for targeted drug delivery in treatment of inflammatory bowel disease. J Pharmacol Exp Ther. 2001;299(2):775-781.

8. Nakase H, Okazaki K, Tabata Y, et al. An oral drug delivery system targeting immune-regulating cells ameliorates mucosal injury in trinitrobenzene sulfonic acid-induced colitis. J Pharmacol Exp Ther. 2001; 297(3):1122-1128.

9. Nakase H, Okazaki K, Tabata Y, et al. Development of an oral drug delivery system targeting immune-regulating cells in experimental inflammatory bowel disease: a new therapeutic strategy. J Pharmacol Exp Ther. 2000;292(1):15-21.

10. Burey P, Bhandari BR, Howes T, Gidley MJ. Hydrocolloid gel particles: formation, characterization, and application. Crit Rev Food Sci Nutr. 2008;48(5):361-377.

11. Li Y, Rodrigues J, Tomas H. Injectable and biodegradable hydrogels: gelation, biodegradation and biomedical applications. Chem Soc Rev 2012;41(6):2193-2221.

12. Wang Y, Kim H-J, Vunjak-Novakovic G, Kaplan DL. Stem cell-based tissue engineering with silk biomaterials. Biomaterials. 2006;27(36): 6064-6082.

13. Shao W, He J, Han Q, et al. A biomimetic multilayer nanofiber fabric fabricated by electrospinning and textile technology from polylactic acid and Tussah silk fibroin as a scaffold for bone tissue engineering. Mater Sci Eng C Mater Biol Appl. 2016;67:599-610.

14. Sun W, Motta A, Shi Y, et al. Co-culture of outgrowth endothelial cells with human mesenchymal stem cells in silk fibroin hydrogels promotes angiogenesis. Biomed Mater. 2016;11(3):035009.

15. Sharma S, Bano S, Ghosh AS, et al. Silk fibroin nanoparticles support in vitro sustained antibiotic release and osteogenesis on titanium surface. Nanomedicine. 2016;12(5):1193-1204.

16. Liu Q, Liu H, Fan Y. Preparation of silk fibroin carriers for controlled release. Microsc Res Tech. Epub 2015 Dec 6.

17. Wenk E, Wandrey AJ, Merkle HP, Meinel L. Silk fibroin spheres as a platform for controlled drug delivery. J Control Release. 2008; 132(1):26-34.

18. Hino T, Tanimoto M, Shimabayashi S. Change in secondary structure of silk fibroin during preparation of its microspheres by spray-drying and exposure to humid atmosphere. J Colloid Interface Sci. 2003;266(1): 68-73.

19. Kundu J, Chung YI, Kim YH, Tae G, Kundu SC. Silk fibroin nanoparticles for cellular uptake and control release. Int J Pharm. 2010; 388(1-2):242-250.

20. Zhang Y-Q, Shen W-D, Xiang R-L, Zhuge L-J, Gao W-J, Wang W-B. Formation of silk fibroin nanoparticles in water-miscible organic solvent and their characterization. J Nanopart Res. 2006;9(5): 885-900.

21. Kim E-D, Bayaraa T, Shin E-J, Hyun C-K. Fibroin-derived peptides stimulate glucose transport in normal and insulin-resistant 3T3-L1 adipocytes. Biol Pharm Bull. 2009;32(3):427-433.

22. Yamada H, Igarashi Y, Takasu Y, Saito H, Tsubouchi K. Identification of fibroin-derived peptides enhancing the proliferation of cultured human skin fibroblasts. Biomaterials. 2004;25(3):467-472.

23. Kim SY, Sohn EJ, Kim DW, et al. Transduced PEP-1-FK506BP ameliorates atopic dermatitis in NC/Nga mice. J Invest Dermatol. 2011;131(7): 1477-1485.
24. Lozano-Perez AA, Rodriguez-Nogales A, Ortiz-Cullera V, et al. Silk fibroin nanoparticles constitute a vector for controlled release of resveratrol in an experimental model of inflammatory bowel disease in rats. Int J Nanomedicine. 2014;9:4507-4520.

25. Kimura T, Yamada H, Tsubouchi K, Doi K. Accelerating effects of silk fibroin on wound healing in hairless descendants of Mexican hairless dogs. J Appl Sci Res. 2007;3(11):1306-1314.

26. Martinez-Mora C, Mrowiec A, Garcia-Vizcaino EM, Alcaraz A, Cenis JL, Nicolas FJ. Fibroin and sericin from Bombyx mori silk stimulate cell migration through upregulation and phosphorylation of c-Jun. PLoS One. 2012;7(7):e42271.

27. Pineton de Chambrun G, Peyrin-Biroulet L, Lemann M, Colombel JF. Clinical implications of mucosal healing for the management of IBD. Nat Rev Gastroenterol Hepatol. 2010;7(1):15-29.

28. Arosio D, Manzoni L, Araldi EM, Scolastico C. Cyclic RGD functionalized gold nanoparticles for tumor targeting. Bioconjug Chem. 2011; 22(4):664-672.

29. Nielsen OH, Seidelin JB, Munck LK, Rogler G. Use of biological molecules in the treatment of inflammatory bowel disease. J Intern Med. 2011; 270(1):15-28.

30. Singh D, Srivastava S, Pradhan M, Kanwar JR, Singh MR. Inflammatory bowel disease: pathogenesis, causative factors, issues, drug treatment strategies, and delivery approaches. Crit Rev Ther Drug Carrier Syst. 2015;32(3):181-214.

31. Nielsen $\mathrm{OH}$. New strategies for treatment of inflammatory bowel disease. Front Med. 2014;1:3

32. Ajisawa A. Dissolution of silk fibroin with calciumchloride/ethanol aqueous solution. Studies on the dissolution of silk fibroin. J Seric Sci Jpn. 1998;67(2):91-94.

33. Zhang Y-Q, Shen W-D, Xiang R-L, Zhuge L-J, Gao W-J, Wang W-B. Formation of silk fibroin nanoparticles in water-miscible organic solvent and their characterization. J Nanopart Res. 2007;9(5): 885-900.

34. Uchida E, Uyama Y, Ikada Y. Sorption of low-molecular-weight anions into thin polycation layers grafted onto a film. Langmuir. 1993;9(4): $1121-1124$.

35. Noel S, Liberelle B, Robitaille L, De Crescenzo G. Quantification of primary amine groups available for subsequent biofunctionalization of polymer surfaces. Bioconjug Chem. 2011;22(8):1690-1699.

36. Hemmila I. Fluoroimmunoassays and immunofluorometric assays. Clin Chem. 1985;31(3):359-370.

37. Camuesco D, Peran L, Comalada M, et al. Preventative effects of lactulose in the trinitrobenzenesulphonic acid model of rat colitis. Inflamm Bowel Dis. 2005;11(3):265-271.

38. Arribas B, Suarez-Pereira E, Ortiz Mellet C, et al. Di-D-fructose dianhydride-enriched caramels: effect on colon microbiota, inflammation, and tissue damage in trinitrobenzenesulfonic acid-induced colitic rats. J Agric Food Chem. 2010;58(10):6476-6484.

39. Krawisz JE, Sharon P, Stenson WF. Quantitative assay for acute intestinal inflammation based on myeloperoxidase activity. Assessment of inflammation in rat and hamster models. Gastroenterology. 1984;87(6): 1344-1350.

40. Anderson ME. Determination of glutathione and glutathione disulfide in biological samples. Methods Enzymol. 1985;113:548-555.

41. Cao Z, Chen X, Yao J, Huang L, Shao Z. The preparation of regenerated silk fibroin microspheres. Soft Matter. 2007;3(7):910.

42. Zhu H, Li YR. Oxidative stress and redox signaling mechanisms of inflammatory bowel disease: updated experimental and clinical evidence. Exp Biol Med. 2012;237(5):474-480.

43. Algieri F, Zorrilla P, Rodriguez-Nogales A, et al. Intestinal antiinflammatory activity of hydroalcoholic extracts of Phlomis purpurea $\mathrm{L}$. and Phlomis lychnitis L. in the trinitrobenzenesulphonic acid model of rat colitis. J Ethnopharmacol. 2013;146(3):750-759.

44. Algieri F, Rodriguez-Nogales A, Garrido-Mesa N, et al. Intestinal anti-inflammatory activity of the Serpylli herba extract in experimental models of rodent colitis. J Crohns Colitis. 2014;8(8): 775-788. 
45. Camuesco D, Comalada M, Rodriguez-Cabezas ME, et al. The intestinal anti-inflammatory effect of quercitrin is associated with an inhibition in iNOS expression. Br J Pharmacol. 2004;143(7):908-918.

46. Kim YS, Ho SB. Intestinal goblet cells and mucins in health and disease: recent insights and progress. Curr Gastroenterol Rep. 2010; 12(5):319-330.

47. Bravata I, Allocca M, Fiorino G, Danese S. Integrins and adhesion molecules as targets to treat inflammatory bowel disease. Curr Opin Pharmacol. 2015;25:67-71.

48. Sanchez de Medina F, Galvez J, Romero JA, Zarzuelo A. Effect of quercitrin on acute and chronic experimental colitis in the rat. J Pharmacol Exp Ther. 1996;278(2):771-779.

49. Garcia-Ramallo E, Marques T, Prats N, Beleta J, Kunkel SL, Godessart N. Resident cell chemokine expression serves as the major mechanism for leukocyte recruitment during local inflammation. J Immunol. 2002; 169(11):6467-6473.

50. Veljaca M, Lesch CA, Pllana R, Sanchez B, Chan K, Guglietta A. Bpc-15 reduces trinitrobenzene sulfonic acid-induced colonic damage in rats. J Pharmacol Exp Ther. 1995;272(1):417-422.
51. Kinoshita K, Horiguchi K, Fujisawa M, et al. Possible involvement of muscularis resident macrophages in impairment of interstitial cells of Cajal and myenteric nerve systems in rat models of TNBS-induced colitis. Histochem Cell Biol. 2007;127(1):41-53.

52. Watanabe T, Higuchi K, Hamaguchi M, et al. Monocyte chemotactic protein-1 regulates leukocyte recruitment during gastric ulcer recurrence induced by tumor necrosis factor-alpha. Am J Physiol Gastrointest Liver Physiol. 2004;287(4):G919-G928.

53. Leon AJ, Gomez E, Garrote JA, et al. High levels of proinflammatory cytokines, but not markers of tissue injury, in unaffected intestinal areas from patients with IBD. Mediators Inflamm. 2009;2009:580450.

54. Tytgat KM, van der Wal JW, Einerhand AW, Buller HA, Dekker J. Quantitative analysis of MUC2 synthesis in ulcerative colitis. Biochem Biophys Res Commun. 1996;224(2):397-405.

55. Podolsky DK, Gerken G, Eyking A, Cario E. Colitis-associated variant of TLR2 causes impaired mucosal repair because of TFF3 deficiency. Gastroenterology. 2009;137(1):209-220.

56. Neurath MF. New targets for mucosal healing and therapy in inflammatory bowel diseases. Mucosal Immunol. 2014;7(1):6-19.
International Journal of Nanomedicine

\section{Publish your work in this journal}

The International Journal of Nanomedicine is an international, peerreviewed journal focusing on the application of nanotechnology in diagnostics, therapeutics, and drug delivery systems throughout the biomedical field. This journal is indexed on PubMed Central, MedLine, CAS, SciSearch $\AA$, Current Contents ${ }^{\circledR} /$ Clinical Medicine,

\section{Dovepress}

Journal Citation Reports/Science Edition, EMBase, Scopus and the Elsevier Bibliographic databases. The manuscript management system is completely online and includes a very quick and fair peer-review system, which is all easy to use. Visit http://www.dovepress.com/ testimonials.php to read real quotes from published authors. 